\title{
Recurrence for values of the zeta function
}

\author{
Marc Prévost \\ Univ Lille Nord de France, F-59000 Lille, France \\ ULCO, LMPA J. Liouville, B.P. 699, F-62228 Calais \\ CNRS, FR 2956
}

\begin{abstract}
Using the Padé approximation of the exponential function, we obtain a general recurrence relation for values of the zeta function which contains, as particular cases, many of relations already proved. Applications to Bernoulli polynomials are given. At last, we derive some new recurrence relations with gap of length 4 for zeta numbers.
\end{abstract}

MSC: 11B68 41A21

Key words: Zeta function, Padé approximants

\section{Introduction and motivation}

The Riemann zeta-function $\zeta(s)$ and the Hurwitz (or generalized) zeta function $\zeta(s, a)$ are defined by

$$
\zeta(s):=\sum_{k=1}^{\infty} \frac{1}{k^{s}}=\frac{1}{1-2^{-s}} \sum_{k=1}^{\infty} \frac{1}{(2 k-1)^{s}},(\Re(s)>1)
$$

and

$$
\zeta(s, a):=\sum_{k=0}^{\infty} \frac{1}{(k+a)^{s}},\left(\Re(s)>1 ; a \notin \mathbb{Z}^{-}\right) .
$$

The series for Hurwitz zeta function converges absolutely for $\Re(s)=\sigma>1$ and the convergence is uniform in the half plane $\sigma \geq \sigma_{0}>1$. So $\zeta(s, a)$ is analytic in the half-plane $\Re(s)=\sigma>1$. It can be represented by the following integral

$$
\zeta(s, a)=\frac{1}{\Gamma(s)} \int_{0}^{\infty} \frac{x^{s-1} e^{-a x}}{1-e^{-x}} d x,(\Re(s)>1 ; \Re(a)>0)
$$

and can be continued meromorphically to the whole complex $s$-plane with a simple pole at $s=1$ where the residue is 1 , by the contour integral representation

$$
\zeta(s, a)=-\frac{\Gamma(1-s)}{2 i \pi} \int_{C} \frac{(-z)^{s-1} e^{-a z}}{1-e^{-z}} d z,(\Re(s)>1 ; \Re(a)>0)
$$

(the contour $C$ is the Hankel loop i.e. composed of three parts $C_{1}, C_{2}, C_{3}$ where $C_{2}$ is a small circle of radius $\rho$ less than $2 \pi$ centered on the origin and $C_{1}, C_{3}$ are the lines $[ \pm i \rho,+\infty \pm i \rho[)$. From the definition (1.2) of $\zeta(s, a)$,

$$
\begin{aligned}
\zeta(s, a)-\zeta(s, a+p) & =\sum_{k=0}^{p-1} \frac{1}{(k+a)^{s}},(p \in \mathbb{N}) \text { and } \\
\zeta(s, a)-\zeta\left(s, a+\frac{1}{2}\right) & =2^{s} \sum_{k=0}^{\infty} \frac{(-1)^{k}}{(2 a+k)^{s}} .
\end{aligned}
$$

Email address: prevost@lmpa.univ-littoral.fr (Marc Prévost) 
Classical Bernoulli polynomials of degree $n$ in the variable $x$ are usually defined by means of the following generating function $\frac{t}{e^{t}-1} e^{x t}=\sum_{k=0}^{\infty} B_{k}(x) \frac{t^{k}}{k !},(|t|<2 \pi)$.

The connection between $\zeta(s, x)$ for $s=-r \in \mathbb{Z}^{-}$and the Bernoulli polynomials is given as follows:

$$
\zeta(-r, x)=-\frac{B_{r+1}(x)}{r+1},(r \in \mathbb{N}) .
$$

Moreover, for even index, the numbers $\zeta(2 n), n \in \mathbb{N}$, can be expressed in terms of Bernoulli numbers $B_{2 n}$ defined by $B_{k}:=B_{k}(0)$, as follows:

$$
\zeta(2 n)=(-1)^{n+1} \frac{(2 \pi)^{2 n}}{2(2 n) !} B_{2 n},(n \in \mathbb{N}),
$$

and for odd index

$$
\zeta(2 n+1)=(-1)^{n+1} \frac{(2 \pi)^{2 n+1}}{2(2 n+1) !} \int_{0}^{1} B_{2 n+1}(t) \cot (\pi t) d t,(n \in \mathbb{N}) .
$$

In [14], the authors prove many identities involving the zeta function. Let us recall some of these formulas:

$$
\begin{gathered}
\sum_{k=2}^{\infty} \zeta(k, 2)=1 \\
\zeta(s)=1+\frac{1}{s-1}-\sum_{k=1}^{\infty} \frac{(s)_{k}}{(k+1) !} \zeta(s+k, 2), \\
\left(1-2^{1-s}\right) \zeta(s)=\sum_{k=1}^{\infty} \frac{(s)_{k}}{(k) !} \frac{\zeta(s+k)}{2^{s+k}} \\
\zeta(s, a)=a^{-s}\left(\frac{1}{2}+\frac{a}{s-1}\right)+\frac{1}{2} \sum_{k=1}^{\infty} \frac{(-1)^{k-1} k(s)_{k+1}}{(k+2) !} \zeta(s+k+1, a), \\
\sum_{k=2}^{\infty} \frac{k-1}{2^{k}} \zeta(k, 2)=\frac{\pi^{2}}{8}-1 .
\end{gathered}
$$

The previous relations derive from the following identity

$$
\sum_{k=0}^{\infty} \frac{(s)_{k}}{k !} \zeta(s+k, a) t^{k}=\zeta(s, a-t),(|t|<|a|),
$$

easily proved by using the binomial expansion $\sum_{k=0}^{\infty} \frac{(s)_{k}}{k !} z^{k}=(1-z)^{-s},(|z|<1)$ in the definition (1.3) of zeta function, the Pochhammer symbol $(s)_{k}$ is being defined as

$$
(s)_{k}=s(s+1) \cdots(s+k-1)=\frac{\Gamma(s+k)}{\Gamma(s)}(\text { if } k \geq 1) ; 1(\text { if } k=0) .
$$

In this article, we are interesting with recurrence formula for the values of the $\zeta$-function on the set $\{s, s+1, s+2, \ldots\}, s \in \mathbb{C}$. The core of the paper is Section 3, Theorem 1. Using Padé approximants to the exponential function in the integral expression (1.3) of $\zeta(s, a)$, we prove a recurrence relation depending on five parameters $n, m, p, a, s$ where $n$ and $m$ are respectively the degree of the numerator and the degree of the denominator of the Padé approximant used to approximate the function $e^{t}$ and $p, a, s$ are some complex numbers.

For negative integer value of the complex number $s$, recurrence relations between Bernoulli polynomials are recovered. 
The paper is organized as follows. In the next section, we recall the definition of Padé approximants to a general series and give its expression for the case of the exponential function. In Section 3, we apply Padé approximation to prove a general linear recurrence relation for the zeta numbers and in Section 6 we prove that many known linear recurrence relations are particular cases. At last, an arithmetical property of the linear combination of $\zeta$-values is proved.

\section{Padé approximant}

In this section, we recall the definition of Padé approximation to general series and their expression in the case of the exponential function.

Given a function $f$ with a Taylor expansion $f(t)=\sum_{i=0}^{\infty} c_{i} t^{i}$ in a neighborhood of the origin, a Padé approximant denoted $[n, m]_{f}$ to $f$ is a rational fraction of degree $n$ (resp. $m$ ) for the numerator (resp. the denominator):

$$
[n, m]_{f}(t)=\frac{\alpha_{0}+\alpha_{1} t+\cdots+\alpha_{n} t^{n}}{\beta_{0}+\beta_{1} t+\cdots+\beta_{m} t^{m}}
$$

whose Taylor expansion agrees with those of $f$ as far as possible:

$$
\sum_{i=0}^{\infty} c_{i} t^{i}-\frac{\alpha_{0}+\alpha_{1} t+\cdots+\alpha_{n} t^{n}}{\beta_{0}+\beta_{1} t+\cdots+\beta_{m} t^{m}}=O\left(t^{m+n+1}\right)
$$

In the general case, the resulting linear system has unique solutions $\alpha_{i}, \beta_{i}$; (see, e.g., [2]).

Padé approximation is related with convergence acceleration [7], continued fractions [3, 10], orthogonal polynomials and with quadrature formulas [9]. Moreover the denominators of Padé approximants satisfy a three terms recurrence relationship $[5,6]$ and this property allowed finding another proof of the irrationality of $\zeta(2)$ and $\zeta(3)$ (see [13]).

If $f(t)=e^{t}$ then

$$
[n, m]_{f}(t):=\frac{P^{(n, m)}(t)}{Q^{(n, m)}(t)}=\frac{{ }_{1} F_{1}(-n,-m-n, t)}{{ }_{1} F_{1}(-m,-m-n,-t)}=\frac{\sum_{j=0}^{n} \frac{(-n)_{j}}{(-m-n)_{j}} \frac{t^{j}}{j !}}{\sum_{j=0}^{m} \frac{(-m)_{j}}{(-m-n)_{j}} \frac{(-t))^{j}}{j !}}=\frac{\sum_{j=0}^{n}\left(\begin{array}{c}
m+n-j \\
m
\end{array}\right) \frac{t^{j}}{j !}}{\sum_{j=0}^{m}\left(\begin{array}{c}
m+n-j \\
n
\end{array}\right) \frac{(-t)^{j}}{j !}},
$$

where the hypergeometric series ${ }_{1} F_{1}(a, b, z)$ is defined as ${ }_{1} F_{1}(a, b, z):=\sum_{k=0}^{\infty} \frac{(a)_{k}}{(b)_{k}} \frac{z^{k}}{k !}$.

In what follows, we write $[n, m](t)$ for the Padé approximant to $e^{t}$. Let us set

$$
\alpha_{j}^{(n, m)}:=\left(\begin{array}{c}
m+n-j \\
m
\end{array}\right) \frac{1}{j !}, \quad 0 \leq j \leq n, \quad \beta_{j}^{(n, m)}:=\left(\begin{array}{c}
m+n-j \\
n
\end{array}\right) \frac{(-1)^{j}}{j !}, \quad 0 \leq j \leq m .
$$

The polynomials $P^{(n, m)}, Q^{(n, m)}$ are then given by

$$
P^{(n, m)}(t)=\sum_{j=0}^{n} \alpha_{j}^{(n, m)} t^{j}, \quad Q^{(n, m)}(t)=\sum_{j=0}^{m} \beta_{j}^{(n, m)} t^{j} .
$$

The error term is defined by $R^{(n, m)}(t):=e^{t}-[n, m](t)=e^{t}-\frac{P^{(n, m)}(t)}{Q^{(n, m)}(t)}$ and satisfies

$$
\begin{aligned}
& R^{(n, m)}(t)=\frac{(-1)^{m}}{Q^{(n, m)}(t)} \frac{t^{m+n+1}}{n ! m !} \int_{0}^{1} x^{m}(1-x)^{n} e^{x t} d x \\
& =\frac{(-1)^{m}}{Q^{(n, m)}(t)} \sum_{j=0}^{\infty} \frac{1}{(m+n+j+1) !}\left(\begin{array}{c}
m+j \\
m
\end{array}\right) t^{j+m+n+1}=O\left(t^{n+m+1}\right) .
\end{aligned}
$$




\section{Recurrence relation}

\subsection{General formula}

Let us recall one method to derive the formula (1.8). In the expression of $\zeta(s, a-t)$,

$$
\zeta(s, a-t)=\frac{1}{\Gamma(s)} \int_{0}^{\infty} \frac{x^{s-1} e^{-(a-t) x}}{1-e^{-x}} d x=\frac{1}{\Gamma(s)} \int_{0}^{\infty} \frac{x^{s-1} e^{-a x} e^{t x}}{1-e^{-x}} d x
$$

we replace $e^{t x}$ by its Taylor expansion around $t=0$ to get:

$$
\begin{aligned}
\zeta(s, a-t) & =\frac{1}{\Gamma(s)} \sum_{k=0}^{\infty} \frac{t^{k}}{k !} \int_{0}^{\infty} \frac{x^{s+k-1} e^{-a x}}{1-e^{-x}} d x=\frac{1}{\Gamma(s)} \sum_{k=0}^{\infty} \frac{t^{k}}{k !} \Gamma(s+k) \zeta(s+k, a) \\
& =\sum_{k=0}^{\infty} \frac{t^{k}}{k !}(s)_{k} \zeta(s+k, a) .
\end{aligned}
$$

In this section, the main idea is to replace in $\zeta(s, a-t)$, the exponential function $e^{t x}$, not by its taylor expansion around $t=0$ but by its Padé approximant $[n, m](t)$. This gives the following main result.

Theorem 1. For $n, m \in \mathbb{N}, s \in \mathbb{C} \backslash D_{n, m}$ where $D_{n, m}:=\{-n-m, 1-\max (n, m), \cdots,-1,0,1\}$, for $(p, a) \in \mathbb{C}^{2}$, let us define the quantity $A_{(n, m)}^{(p)}(s, a)$ by

$$
\begin{aligned}
A_{(n, m)}^{(p)}(s, a) & :=\left(\begin{array}{c}
n+m \\
n
\end{array}\right)^{-1}\left(\sum_{j=0}^{m}\left(\begin{array}{c}
n+m-j \\
n
\end{array}\right) p^{j} \frac{(s)_{j}}{j !} \zeta(s+j, a+p)-\sum_{j=0}^{n}\left(\begin{array}{c}
n+m-j \\
m
\end{array}\right)(-p)^{j} \frac{(s)_{j}}{j !} \zeta(s+j, a)\right) \\
& =\sum_{j=0}^{m} \frac{(-m)_{j}}{(-n-m)_{j}} p^{j} \frac{(s)_{j}}{j !} \zeta(s+j, a+p)-\sum_{j=0}^{n} \frac{(-n)_{j}}{(-n-m)_{j}}(-p)^{j} \frac{(s)_{j}}{j !} \zeta(s+j, a) .
\end{aligned}
$$

If $\Re(a)>0$ and $\Re(a+p)>0$, then

$$
A_{(n, m)}^{(p)}(s, a)=(-1)^{n+1} p^{m+n+1} \frac{(s)_{m+n+1}}{(n+m) !} \int_{0}^{1} x^{m}(1-x)^{n} \zeta(s+m+n+1, a+p x) d x .
$$

Proof. Considering the remainder term for Padé approximant to $e^{-p t}(2.1)$,

$$
e^{-p t} Q^{(n, m)}(-p t)-P^{(n, m)}(-p t)=(-1)^{n+1} p^{m+n+1} \frac{t^{m+n+1}}{n ! m !} \int_{0}^{1} x^{m}(1-x)^{n} e^{-x p t} d x,
$$

for $\Re(a)>0, \Re(a+p)>0$ and $\Re(s)>1$, we multiply the two handside members by $\frac{t^{s-1} e^{-a t}}{1-e^{-t}}$ and integrate for $t \in[0, \infty]$ :

$$
\begin{aligned}
& \sum_{j=0}^{m} \beta_{j}^{(n, m)}(-p)^{j} \int_{0}^{+\infty} e^{-p t} \frac{t^{j+s-1} e^{-a t}}{1-e^{-t}} d t-\sum_{j=0}^{n} \alpha_{j}^{(n, m)}(-p)^{j} \int_{0}^{+\infty} \frac{t^{j+s-1} e^{-a t}}{1-e^{-t}} d t \\
= & \frac{(-1)^{n+1} p^{m+n+1}}{n ! m !} \int_{0}^{+\infty} \frac{t^{s-1} e^{-a t}}{1-e^{-t}} t^{m+n+1} d t \int_{0}^{1} x^{m}(1-x)^{n} e^{-x p t} d x .
\end{aligned}
$$

Thus, using the definition $(1.3)$ of $\zeta(s, a)$,

$$
\begin{aligned}
& \sum_{j=0}^{m} \beta_{j}^{(n, m)}(-p)^{j} \Gamma(s+j) \zeta(s+j, a+p)-\sum_{j=0}^{n} \alpha_{j}^{(n, m)}(-p)^{j} \Gamma(s+j) \zeta(s+j, a) \\
= & \frac{(-1)^{n+1}}{n ! m !} p^{m+n+1} \Gamma(s+m+n+1) \int_{0}^{1} x^{m}(1-x)^{n} d x \int_{0}^{+\infty} \frac{t^{s+m+n}}{1-e^{-t}} e^{-(a+p x) t} d t \\
= & \frac{(-1)^{n+1}}{n ! m !} p^{m+n+1} \Gamma(s+m+n+1) \int_{0}^{1} x^{m}(1-x)^{n} \zeta(s+m+n+1, a+p x) d x,
\end{aligned}
$$


which proves the Theorem 1, after division by $\Gamma(s)\left(\begin{array}{c}n+m \\ n\end{array}\right)$ for $\Re(s)>1$. (Note that the interversion of the signs sum is motivated by the inequality

$\left\|x^{m}(1-x)^{n} \frac{t^{s+m+n}}{1-e^{-t}} e^{-(a+p x) t}\right\| \leq t^{\Re(s)+m+n-1} e^{-\min \{\Re(a), \Re(a+p)\} t}$ which is integrable on $(t, x) \in$ $[0, \infty] \times[0,1])$.

Now, for fixed $a$ and $p$ such that $\Re(a)>0, \Re(a+p)>0$, the two functions of the variable $s$, $A_{(n, m)}^{(p)}(s, a)$ and $(-1)^{n+1} p^{m+n+1} \frac{(s)_{m+n+1}}{(n+m) !} \int_{0}^{1} x^{m}(1-x)^{n} \zeta(s+m+n+1, a+p x) d x$ are analytic on $s \in \mathbb{C} \backslash D_{n, m}$ and are equal for $\Re(s)>1$, so the equality (3.3) is valid for all complex number $s$ in $\mathbb{C} \backslash D_{n, m}$.

Remark 1. The conditions $\Re(a)>0$ and $\Re(a+p)>0$ are not restrictive since $\zeta(s, a)=\zeta(s, a+k)+\sum_{i=0}^{k-1} \frac{1}{(i+a)^{s}}$, and $\zeta(s, a+k)$ will satisfy $\Re(a+k)>0$ for suitable integer $k$.

\subsection{Convergence}

Now, we are interesting with the convergence of $A_{(n, m)}^{(p)}(s, a)$ when $n$ or $m$ tend to infinity.

Lemma 1. If $|p| \leq|a|,|p| \leq|a+p|, \Re(a)>0$ and $\Re(a+p)>0$ then $\forall s \in \mathbb{C} \backslash \mathbb{Z}^{-} \backslash\{1\}$

$$
\left|\int_{0}^{1} x^{m}(1-x)^{n} \zeta(s+m+n+1, a+p x) d x\right| \leq C \frac{1}{|a+p|^{m}} \frac{1}{|a|^{n}},
$$

where $C$ is some constant independent of $m$ and $n$.

Proof. First we will prove that for fixed $c \in \mathbb{C}$ with $\Re(c)>0$ we have $\lim _{\Re(z) \rightarrow+\infty} c^{z} \zeta(z, c)=1$.

Actually, $\left|c^{z} \zeta(z, c)-1\right| \leq \sum_{k=1}^{\infty}\left|\frac{c}{c+k}\right|^{\Re(z)} \exp \left(-\Im(z) \operatorname{Arg}\left(\frac{c}{c+k}\right)\right) \leq e^{|\Im(z)||\operatorname{Arg}(c)|} \sum_{k=1}^{\infty}\left|\frac{c}{c+k}\right|^{\Re(z)}$ which tends to 0 when $\Re(z)$ tends to infinity.

Now, if we take $c=a+p x$, then $\forall x \in[0,1]$, from the hypothesis, $\Re(a+p x)>0$ which implies $\forall k \geq 1, \forall x \in[0,1],\left|\frac{a+p x}{a+p x+k}\right|<1$. Thus $\left|\zeta(s+m+n+1, a+p x)(a+p x)^{s+m+n+1}\right|$ is bounded by some constant $K$ independent of $x \in[0,1], m \in \mathbb{N}$ and $n \in \mathbb{N}$.

This implies

$$
\begin{aligned}
& \left|\int_{0}^{1} x^{m}(1-x)^{n} \zeta(s+m+n+1, a+p x) d x\right| \\
& =\left|\int_{0}^{1} \frac{x^{m}(1-x)^{n}}{(a+p x)^{m+n}} \zeta(s+m+n+1, a+p x)(a+p x)^{s+m+n+1} \frac{d x}{(a+p x)^{s+1}}\right| \\
& \leq K \int_{0}^{1}\left(\frac{x}{|a+p x|}\right)^{m}\left(\frac{1-x}{|a+p x|}\right)^{n} \frac{d x}{\left|(a+p x)^{s+1}\right|} \\
& \leq K\left(\max _{0 \leq x \leq 1} \frac{x}{|a+p x|}\right)^{m}\left(\max _{0 \leq x \leq 1} \frac{1-x}{|a+p x|}\right)^{n} \int_{0}^{1} \frac{d x}{\left|(a+p x)^{s+1}\right|} .
\end{aligned}
$$

Let us assume that $|p| \leq|a|$ and calculate the derivative of $\phi(x):=\frac{x^{2}}{|a+p x|^{2}}$.

$\phi^{\prime}(x)=\frac{x\left(2|a|^{2}+(\bar{a} p+a \bar{p}) x\right)}{|a+p x|^{4}} \geq 0, \forall x \in[0,1]$, since $|(\bar{a} p+a \bar{p}) x| \leq 2|a||p| x \leq 2|a||p| \leq 2|a|^{2}$. Thus $\phi$ is increasing on $[0,1]$ and $\max _{0 \leq x \leq 1} \frac{x}{|a+p x|}=\frac{1}{|a+p|}$.

With the change of variable $X=1-x$, we deduce that $|p| \leq|a+p| \Rightarrow \max _{0 \leq x \leq 1} \frac{1-x}{|a+p x|}=$ $\max _{0 \leq X \leq 1} \frac{X}{|a+p-p X|}=\frac{1}{|a|}$ and Lemma 1 is proved with $C=K \cdot \int_{0}^{1} \frac{d x}{\left|(a+p x)^{s+1}\right|}$.

We are now in position to prove the convergence of the general formula (3.1). 
Theorem 2. If $|p| \leq|a|,|p|<|a+p|, \Re(a)>0$ and $\Re(a+p)>0$ then

$$
\begin{array}{r}
\forall s \in \mathbb{C} \backslash \mathbb{Z}^{-} \backslash\{1\}, \lim _{m \rightarrow \infty} A_{(n, m)}^{(p)}(s, a)=0 . \\
\text { If }|p|<|a|,|p| \leq|a+p|, \Re(a)>0 \text { and } \Re(a+p)>0 \text { then } \\
\forall s \in \mathbb{C} \backslash \mathbb{Z}^{-} \backslash\{1\}, \lim _{n \rightarrow \infty} A_{(n, m)}^{(p)}(s, a)=0 .
\end{array}
$$

Proof. In Theorem 1, we proved

$$
A_{(n, m)}^{(p)}(s, a)=(-1)^{n+1} p^{m+n+1} \frac{(s)_{m+n+1}}{(n+m) !} \int_{0}^{1} x^{m}(1-x)^{n} \zeta(s+m+n+1, a+p x) d x .
$$

Lemma 1 implies $\left|A_{(n, m)}^{(p)}(s, a)\right| \leq C|p|^{m+n+1} \frac{(|s|)_{m+n+1}}{(n+m) !} \frac{1}{|a+p|^{m}} \frac{1}{|a|^{n}}$. If $|p| \leq|a|$ and $|p|<|a+p|$, when $m$ tends to infinity, the quotient $|p|^{m+n+1} \frac{1}{|a+p|^{m}} \frac{1}{|a|^{n}}$ tend to 0 .

Moreover, the term $\frac{(|s|)_{m+n+1}}{(n+m) !}$ is less than to $\frac{(m+n+|s|)^{|s|}}{\Gamma(|s|)}$, and thus the product $\frac{(|s|)_{m+n+1}}{(n+m) !}|p|^{m+n+1} \frac{1}{|a+p|^{m}} \frac{1}{|a|^{n}}$ tends to 0 when $n$ tends to infinity.

The proof for the case $|p|<|a|,|p| \leq|a+p|$ is similar.

Remark 2. If $n=0$ then for all integer $m, \forall s \in \mathbb{C} \backslash D_{m}$ where $D_{m}:=\{-m,-m+1, \cdots,-2,-1,0,1\}, \forall(p, a) \in \mathbb{C}^{2} / \Re(a)>0, \Re(a+p)>0$, then Theorem 1 reduces to

$$
\sum_{j=0}^{m} p^{j} \frac{(s)_{j}}{j !} \zeta(s+j, a+p)=\zeta(s, a)-p^{m+1} \frac{(s)_{m+1}}{m !} \int_{0}^{1} x^{m} \zeta(s+m+1, a+p x) d x .
$$

When $|p|<|a+p|, \lim _{m \rightarrow \infty} A_{(0, m)}^{(p)}(s, a)=\lim _{m \rightarrow \infty}\left(\sum_{j=0}^{m} p^{j} \frac{(s)_{j}}{j !} \zeta(s+j, a+p)\right)-\zeta(s, a)=0$ and then

$$
\forall s \in \mathbb{C} \backslash \mathbb{Z}^{-} \backslash\{1\}, \sum_{j=0}^{\infty} \frac{(s)_{j}}{j !} \zeta(s+j, a+p) p^{j}=\zeta(s, a) \quad(|p|<|a+p|)
$$

which equivalent to the formula (1.8).

Thus the above formula (3.6) can be considered as the finite version of (1.8) ([14] p.144, [16])

\section{Applications to Bernoulli polynomials}

We recall that Bernoulli polynomials are defined by $\frac{t}{e^{t}-1} e^{x t}=\sum_{k=0}^{\infty} B_{k}(x) \frac{t^{k}}{k !}$, $(|t|<2 \pi)$.

The first polynomials are $B_{0}(x)=1, B_{1}(x)=x-1 / 2, B_{2}(x)=x^{2}-x+1 / 6$.

From the generating function of the Bernoulli polynomials, it is easily proved that

$$
B_{n}(1-x)=(-1)^{n} B_{n}(x), B_{n}(x+1)-B_{n}(x)=n x^{n-1}, B_{n}(x+y)=\sum_{k=0}^{n}\left(\begin{array}{l}
n \\
k
\end{array}\right) B_{k}(x) y^{n-k} .
$$

The classical Bernoulli numbers $B_{n}$ are given by $B_{n}:=B_{n}(0)$.

In 2000, T. Agoh [1] proved a general linear recurrence relation between Bernoulli and Euler polynomials. More recently, in [8], Chen and Sun made use of Zeilberger's algorithm to give new proofs of most of the existing recurrence relations for Bernoulli polynomials. They also derived two new identities which are particular cases of the following theorem. 
Theorem 3. For $r \in \mathbb{N}, r \in[\max (n, m), m+n-1],(m \geq 1, n \geq 1)$

$$
\sum_{j=0}^{m}\left(\begin{array}{c}
n+m-j \\
n
\end{array}\right)\left(\begin{array}{c}
r+1 \\
j
\end{array}\right)(-p)^{j} B_{r+1-j}(a+p)=\sum_{j=0}^{n}\left(\begin{array}{c}
n+m-j \\
m
\end{array}\right)\left(\begin{array}{c}
r+1 \\
j
\end{array}\right) p^{j} B_{r+1-j}(a) .
$$

For $r \in \mathbb{N}, r \geq m+n+1$,

$$
\begin{array}{r}
\sum_{j=0}^{m}\left(\begin{array}{c}
n+m-j \\
n
\end{array}\right)\left(\begin{array}{c}
r+1 \\
j
\end{array}\right)(-p)^{j} B_{r+1-j}(a+p)-\sum_{j=0}^{n}\left(\begin{array}{c}
n+m-j \\
m
\end{array}\right)\left(\begin{array}{c}
r+1 \\
j
\end{array}\right) p^{j} B_{r+1-j}(a) \\
=(-1)^{m} \sum_{k=0}^{r-n-m}\left(\begin{array}{c}
r-n-k \\
m
\end{array}\right)\left(\begin{array}{c}
r+1 \\
k
\end{array}\right) B_{k}(a) p^{r-k+1} .
\end{array}
$$

For $r=m+n$,

$$
\begin{gathered}
\sum_{j=0}^{m}\left(\begin{array}{c}
n+m-j \\
n
\end{array}\right)\left(\begin{array}{c}
m+n+1 \\
j
\end{array}\right)(-p)^{j} B_{m+n+1-j}(a+p)-\sum_{j=0}^{n}\left(\begin{array}{c}
n+m-j \\
m
\end{array}\right)\left(\begin{array}{c}
m+n+1 \\
j
\end{array}\right) p^{j} B_{m+n+1-j}(a) \\
=(-1)^{m} p^{m+n+1} .
\end{gathered}
$$

Proof. For some negative integer $s=-k,(k \in \mathbb{N})$, the connection between $\zeta$-function and Bernoulli polynomials is given by $\zeta(-k, x)=-\frac{B_{k+1}(x)}{k+1}$.

So, in the formula (3.1), we have to consider the sets $\{s+j, 0 \leq j \leq m\} \cap \mathbb{Z}^{-},\{s+j, 0 \leq j \leq n\} \cap \mathbb{Z}^{-}$ and $\{s+m+n+1\} \cap \mathbb{Z}^{-}$.

In (3.1), let us $s=-r$, with $r$ a positive integer $r \geq \max (n, m)$. After simplification, (3.1)

$\sum_{j=0}^{m}\left(\begin{array}{c}n+m-j \\ n\end{array}\right) p^{j} \frac{(s)_{j}}{j !} \zeta(s+j, a+p)-\sum_{j=0}^{n}\left(\begin{array}{c}n+m-j \\ m\end{array}\right)(-p)^{j} \frac{(s)_{j}}{j !} \zeta(s+j, a)=\left(\begin{array}{c}n+m \\ n\end{array}\right) A_{(n, m)}^{(p)}(s, a)$

becomes

$$
\begin{gathered}
\sum_{j=0}^{m}\left(\begin{array}{c}
n+m-j \\
n
\end{array}\right)\left(\begin{array}{c}
r+1 \\
j
\end{array}\right) p^{j}(-1)^{j+1} \frac{B_{r+1-j}(a+p)}{r+1}+\sum_{j=0}^{n}\left(\begin{array}{c}
n+m-j \\
m
\end{array}\right)\left(\begin{array}{c}
r+1 \\
j
\end{array}\right) p^{j} \frac{B_{r+1-j}(a)}{r+1} \\
=\left(\begin{array}{c}
n+m \\
n
\end{array}\right) A_{(n, m)}^{(p)}(-r, a)
\end{gathered}
$$

where $A_{(n, m)}^{(p)}(-r, a)=(-1)^{n+1} p^{m+n+1} \frac{(-r)_{m+n+1}}{(n+m) !} \int_{0}^{1} x^{m}(1-x)^{n} \zeta(-r+m+n+1, a+p x) d x$.

First case: $r \in[\max (n, m), m+n-1]$. We have $\{-r+j, 0 \leq j \leq m\} \subset \mathbb{Z}^{-},\{-r+j, 0 \leq j \leq n\} \subset \mathbb{Z}^{-}$ and $-r+m+n+1 \in \mathbb{Z}^{-}$and thus $\zeta(-r+j, x)=-\frac{B_{r-j+1}(x)}{r-j+1}$ for $j \in[0, \max (m, n)]$. Since $r \in[\max (n, m), m+n-1],(-r)_{m+n+1}=0$ and thus $A_{(n, m)}^{(p)}(-r, a)=0$. After multiplication by $(r+1)$, it arises formula (4.1).

Second case: $r \geq m+n+1$. In that case,

$$
A_{(n, m)}^{(p)}(-r, a)=(-1)^{n+1} p^{m+n+1} \frac{(-r)_{m+n+1}}{(n+m) !} \int_{0}^{1} x^{m}(1-x)^{n} \frac{-B_{r-n-m}(a+p x)}{r-n-m} d x .
$$

Using the following relation $B_{N}(x+y)=\sum_{k=0}^{N}\left(\begin{array}{l}N \\ k\end{array}\right) B_{k}(x) y^{N-k}$, we obtain 


$$
\begin{aligned}
\int_{0}^{1} x^{m}(1-x)^{n} B_{r-n-m}(a+p x) d x & =\sum_{k=0}^{r-n-m}\left(\begin{array}{c}
r-n-m \\
k
\end{array}\right) B_{k}(a) p^{r-n-m-k} \int_{0}^{1} x^{r-n-k}(1-x)^{n} d x \\
& =\sum_{k=0}^{r-n-m}\left(\begin{array}{c}
r-n-m \\
k
\end{array}\right) B_{k}(a) p^{r-n-m-k} \frac{1}{(r-n-k+1)\left(\begin{array}{c}
r-k+1 \\
n
\end{array}\right)}
\end{aligned}
$$

and thus

$$
\begin{aligned}
A_{(n, m)}^{(p)}(-r, a) & =\frac{(-1)^{n} p^{m+n+1}(-r)_{m+n+1}}{(n+m) !(r-n-m)} \sum_{k=0}^{r-n-m}\left(\begin{array}{c}
r-n-m \\
k
\end{array}\right) \frac{1}{(r-n-k+1)\left(\begin{array}{c}
r-k+1 \\
n
\end{array}\right)} B_{k}(a) p^{r-n-m-k} \\
& =\frac{(-1)^{n} p^{m+n+1}(-r)_{m+n+1}}{(n+m) !(r-n-m)} \sum_{k=0}^{r-n-m} \frac{m ! n !(r-m-n) !}{(r+1) !}\left(\begin{array}{c}
r-n-k \\
m
\end{array}\right)\left(\begin{array}{c}
r+1 \\
k
\end{array}\right) B_{k}(a) p^{r-n-m-k} \\
& =\frac{(-1)^{m+1}}{(r+1)}\left(\begin{array}{c}
n+m \\
n
\end{array}\right) \sum_{k=0}^{-1}\left(\begin{array}{c}
r-n-k \\
m
\end{array}\right)\left(\begin{array}{c}
r+1 \\
k
\end{array}\right) B_{k}(a) p^{r-k+1} .
\end{aligned}
$$

From (4.4) we deduce

$$
\begin{aligned}
& \sum_{j=0}^{n}\left(\begin{array}{c}
n+m-j \\
m
\end{array}\right) p^{j}\left(\begin{array}{c}
r+1 \\
j
\end{array}\right) B_{r+1-j}(a)-\sum_{j=0}^{m}\left(\begin{array}{c}
n+m-j \\
n
\end{array}\right)(-p)^{j}\left(\begin{array}{c}
r+1 \\
j
\end{array}\right) B_{r+1-j}(a+p) \\
= & (-1)^{m+1} \sum_{k=0}^{r-n-m}\left(\begin{array}{c}
r-n-k \\
m
\end{array}\right)\left(\begin{array}{c}
r+1 \\
k
\end{array}\right) B_{k}(a) p^{r-k+1} \text { which is formula (4.2). }
\end{aligned}
$$

Third case: $r=m+n$. In that case,

$A_{(n, m)}^{(p)}(-m-n, a)=\lim _{s \rightarrow-m-n}(-1)^{n+1} p^{m+n+1} \frac{(s)_{m+n+1}}{(n+m) !} \int_{0}^{1} x^{m}(1-x)^{n} \zeta(s+m+n+1, a+p x) d x$.

Since $\lim _{z \rightarrow 1}(z-1) \zeta(z, a+p x)=1$, uniformly on $x \in[0,1]$,

$$
\left(\begin{array}{c}
n+m \\
n
\end{array}\right) A_{(n, m)}^{(p)}(-m-n, a)=(-1)^{n+1} p^{m+n+1} \frac{(-1)^{m+n}(m+n) !}{n ! m !\left(\begin{array}{c}
m+n+1 \\
n
\end{array}\right)(m+1)}=(-1)^{m+1} \frac{p^{m+n+1}}{m+n+1} .
$$

Formula (4.3) follows from

$$
\begin{gathered}
\sum_{j=0}^{m}\left(\begin{array}{c}
n+m-j \\
n
\end{array}\right) p^{j}(-1)^{j+1}\left(\begin{array}{c}
r+1 \\
j
\end{array}\right) \frac{B_{r+1-j}(a+p)}{r+1}-\sum_{j=0}^{n}\left(\begin{array}{c}
n+m-j \\
m
\end{array}\right)(-p)^{j}(-1)^{j+1}\left(\begin{array}{c}
r+1 \\
j
\end{array}\right) \frac{B_{r+1-j}(a)}{r+1} \\
=\left(\begin{array}{c}
n+m \\
n
\end{array}\right) A_{(n, m)}^{(p)}(-r, a) \\
\Rightarrow \sum_{j=0}^{m}\left(\begin{array}{c}
n+m-j \\
n
\end{array}\right) p^{j}(-1)^{j+1}\left(\begin{array}{c}
m+n+1 \\
j
\end{array}\right) \frac{B_{m+n+1-j}(a+p)}{m+n+1}- \\
\sum_{j=0}^{n}\left(\begin{array}{c}
n+m-j \\
m
\end{array}\right)(-p)^{j}(-1)^{j+1}\left(\begin{array}{c}
m+n+1 \\
j
\end{array}\right) \frac{B_{m+n+1-j}(a)}{m+n+1}=(-1)^{m+1} \frac{p^{m+n+1}}{m+n+1} .
\end{gathered}
$$

Remark 3. Theorem 3 has been proved in [11] using the generating function of the Bernoulli polynomials; similar formulas for Euler polynomials and Apostol-type polynomials are also available. 


\section{Expression of $A_{(n, m)}^{(p)}(s, a)$ for $m=\lambda n$.}

In the case $m=\lambda n$, it is possible to prove an integral formula for the linear combination of zeta values defined by $A_{(n, m)}^{(p)}(s, a)$. This expression will imply that a sequence $\left(v_{n}\right)_{n \in \mathbb{N}}$ defined with respect to $A_{(n, \lambda n)}^{(p)}(s, a)$ is totally oscillating and thus gives its speed of convergence to zero.

Definition 1. 1) A sequence $\left(u_{n}\right)$ is called totally monotonic (TM) if there exists a non negative measure $d \mu$ with infinitely many points of increase such that

$$
\forall n \in \mathbb{N}, u_{n}=\int_{0}^{\infty} x^{n} d \mu(x) .
$$

If the support of the measure $d \mu$ is the interval $[0,1 / R]$, then for all $n$ we have $u_{n+1} / u_{n} \leq R$ and $\lim _{n} \frac{u_{n+1}}{u_{n}}=R$. If $R=1$, it is equivalent to

$$
\forall n \in \mathbb{N}, k \in \mathbb{N},(-1)^{k} \Delta^{k}\left(u_{n}\right)>0
$$

where $\Delta^{0}\left(u_{n}\right):=u_{n}$ and $\Delta^{k+1} u_{n}=\Delta^{k} u_{n+1}-\Delta^{k} u_{n}$. (see [15], p. 108).

2) If $(-1)^{n} u_{n}$ is TM, then $\left(u_{n}\right)_{n}$ is called totally oscillating (TO).

Remark 4. If a sequence is TM or TO, it is very easy to accelerate its convergence with for example the epsilon-algorithm [4] or the modified moment method [12].

Theorem 4. Let us set $\lambda=\frac{m}{n},(n \geq 1)$. If $s, a$ and $p$ are positive real numbers, then we have the following integral representation

$$
A_{(n, \lambda n)}^{(p)}(s, a)=(-1)^{n+1} p^{(1+\lambda) n+1} \frac{(s)_{(1+\lambda) n+1}}{(n+\lambda n) !} \int_{0}^{M_{0}} u^{n} \phi(u) d u
$$

with $M_{0}=\frac{\lambda^{\lambda}}{(\lambda+1)^{\lambda+1}} \frac{1}{a(a+p)^{\lambda}}$ if $\lambda>0, M_{0}=\frac{1}{a}$ if $\lambda=0$, and $\phi(u)$ is a positive function depending on $\lambda, s$, a and $p$. Moreover, from the theory of moments $\int_{0}^{M_{0}} u^{n} \phi(u) d u=O\left(M_{0}^{n}\right)$.

Proof. In the expression $\int_{0}^{1} x^{\lambda n}(1-x)^{n} \zeta(s+(\lambda+1) n+1, a+p x) d x$, we replace the $\zeta$-function by its series:

$$
\begin{aligned}
\int_{0}^{1} x^{\lambda n}(1-x)^{n} \zeta(s+(\lambda+1) n+1, a+p x) d x & =\int_{0}^{1} x^{\lambda n}(1-x)^{n} \sum_{k=0}^{\infty} \frac{1}{(k+a+p x)^{s+(\lambda+1) n+1}} d x \\
& =\sum_{k=0}^{\infty} \int_{0}^{1}\left(\frac{x^{\lambda}(1-x)}{(k+a+p x)^{\lambda+1}}\right)^{n} \frac{1}{(k+a+p x)^{s+1}} d x .
\end{aligned}
$$

The hypothesis imply that $a+p x>0$ and $s+(\lambda+1) n+1>1$. So the series $\sum_{k=0}^{\infty} \frac{1}{(k+a+p x)^{s+(\lambda+1) n+1}}$ is normally convergent on $x \in[0,1]$. The interversion of the sign $\sum$ and $\int$ are then allowed. Now, for each integer $k$, we set

$$
\Psi_{k}(x):=\frac{x^{\lambda}(1-x)}{(k+a+p x)^{\lambda+1}} \geq 0, x \in[0,1] .
$$

For $\lambda>0$, the function $\Psi_{k}$ attains its maximum $M_{k}$ at $x_{k}=((\lambda+1)+p /(a+k))^{-1} \in[0,1]$ and

$$
M_{k}:=\max \left\{\Psi_{k}(x), x \in[0,1]\right\}=\frac{\lambda^{\lambda}}{(\lambda+1)^{\lambda+1}} \frac{1}{(k+a)(k+a+p)^{\lambda}}>0 .
$$


If $\lambda=0$, then $\Psi_{k}$ is decreasing on $[0,1]$ and thus $M_{k}=\Psi_{k}(0)=\frac{1}{k+a}>0$.

Note that the $\left(M_{k}\right)_{k \in \mathbb{N}}$ is a decreasing sequence.

The function $\Psi_{k}$ applies the interval $[0,1]$ on $\left[0, M_{k}\right]$ :

$$
0 \leq u=\Psi_{k}(X) \leq M_{k} \Leftrightarrow \Phi_{k,-}(u) \leq X \leq \Phi_{k,+}(u) .
$$

For example, if $\lambda=1$,

$$
\Phi_{k, \pm}(u)=\frac{1-2(a+k) p u \pm \sqrt{1-4(a+k)^{2} u-4(a+k) p u}}{2\left(1+p^{2} u\right)}
$$

It implies that

$$
\begin{aligned}
\sum_{k=0}^{\infty} \int_{0}^{1}\left(\frac{x^{\lambda}(1-x)}{(k+a+p x)^{\lambda+1}}\right)^{n} \frac{1}{(k+a+p x)^{s+1}} d x & =\sum_{k=0}^{\infty} \int_{0}^{M_{k}} u^{n}\left(\frac{d \Phi_{k,-}(u)}{\left(k+a+p \Phi_{k,-}(u)\right)^{s+1}}-\frac{d \Phi_{k,+}(u)}{\left(k+a+p \Phi_{k,+}(u)\right)^{s+1}}\right) \\
& =: \sum_{k=0}^{\infty} \int_{0}^{M_{k}} u^{n} \varphi_{k}(u) d u=\int_{0}^{M_{0}} u^{n} \phi(u) d u,
\end{aligned}
$$

where we have set $\phi(u):=\sum_{k=0}^{\infty} I d_{\left[0, M_{k}\right]}(u) \varphi_{k}(u)$.

The functions $\Phi_{k,-}$ and $\Phi_{k,+}$ are respectively increasing and decreasing, so all the functions $\varphi_{k}$ are positive on $\left[0, M_{k}\right]$, and the function $\phi$ is a positive function on $\left[0, M_{0}\right]$. Thus, for each fixed positive reals numbers $s, a, p$, the sequence $\left(A_{(n, \lambda n)}^{(p)}(s, a) /\left(\frac{p^{(1+\lambda) n+1}(s)_{(1+\lambda) n+1}}{(n+\lambda n) !}\right)\right)_{n \geq 1}$ is a totally oscillating sequence with an integral representation on the interval $\left[0, M_{0}\right]$.

\section{Limit case: $m=\lambda n$ and $n$ tends to infinity}

In this section, we will study Theorem 2 when $m$ and $n$ tends to infinity with $m=\lambda n$. Let us recall the expression for $A_{(n, m)}^{(p)}(s, a)$ :

$$
A_{(n, m)}^{(p)}(s, a)=\sum_{j=0}^{m} \frac{(-m)_{j}}{(-n-m)_{j}} p^{j} \frac{(s)_{j}}{j !} \zeta(s+j, a+p)-\sum_{j=0}^{n} \frac{(-n)_{j}}{(-n-m)_{j}}(-p)^{j} \frac{(s)_{j}}{j !} \zeta(s+j, a) .
$$

Let us assume that $m=\lambda n$ with $n$ going to infinity. If $|p| \leq|a|,|p| \leq|a+p|$, with one equality being strict, then Theorem 2 implies

$$
\forall s \in \mathbb{C} \backslash \mathbb{Z}^{-} \backslash\{1\}, \lim _{n} A_{(n, \lambda n)}^{(p)}(s, a)=0,
$$

and using the following asymptotics, for fixed $j \in \mathbb{N}$

$$
\frac{(-\lambda n)_{j}}{(-n-\lambda n)_{j}} \sim_{n \rightarrow \infty}\left(\frac{\lambda}{\lambda+1}\right)^{j}, \quad \frac{(-n)_{j}}{(-n-\lambda n)_{j}} \sim_{n \rightarrow \infty}\left(\frac{1}{\lambda+1}\right)^{j}
$$

we get

$$
\sum_{j=0}^{\infty}\left(\frac{\lambda}{\lambda+1}\right)^{j} p^{j} \frac{(s)_{j}}{j !} \zeta(s+j, a+p)-\sum_{j=0}^{\infty}\left(\frac{1}{\lambda+1}\right)^{j}(-p)^{j} \frac{(s)_{j}}{j !} \zeta(s+j, a)=0 .
$$

where $\lambda$ is a positive rational number. Then, we are led to the following theorem. 
Theorem 5. If $|p| \leq|a|$ and $|p|<|a+p|$ or $|p|<|a|$ and $|p| \leq|a+p|$ and if $\Re(a)>0$, $\Re(a+p)>0$, then $\forall s \in \mathbb{C} \backslash \mathbb{Z}^{-} \backslash\{1\}, \forall \lambda \in \mathbb{C}, \Re(\lambda)>-1 / 2$,

$$
\sum_{j=0}^{\infty}\left(\frac{\lambda}{\lambda+1}\right)^{j} p^{j} \frac{(s)_{j}}{j !} \zeta(s+j, a+p)=\sum_{j=0}^{\infty}\left(\frac{1}{\lambda+1}\right)^{j}(-p)^{j} \frac{(s)_{j}}{j !} \zeta(s+j, a) .
$$

For the particular value $p=1 / 2$,

$$
\begin{aligned}
\sum_{j=1}^{\infty}\left(\left(\frac{\lambda}{\lambda+1}\right)^{j}-\left(\frac{-1}{\lambda+1}\right)^{j}\right) \frac{(s)_{j}}{2^{j} j !} \zeta(s+j, a) & =2^{s} \sum_{j=0}^{\infty}\left(\frac{\lambda}{\lambda+1}\right)^{j} \frac{(s)_{j}}{j !} \sum_{k=0}^{\infty} \frac{(-1)^{k}}{(2 a+k)^{s+j}} \\
& =2^{s} \sum_{k=0}^{\infty} \frac{(-1)^{k}}{\left(2 a+k-\frac{\lambda}{\lambda+1}\right)^{s}}
\end{aligned}
$$

Moreover, for each integer $p$, if $|a| \geq p$ and $\Re(a)>0$, then

$$
\sum_{j=1}^{\infty} \frac{\lambda^{j}-(-1)^{j}}{(\lambda+1)^{j}} p^{j} \frac{(s)_{j}}{j !} \zeta(s+j, a)=\sum_{k=0}^{p-1}\left(k+a-\frac{p \lambda}{\lambda+1}\right)^{-s} .
$$

Proof. If $\Re(\lambda)>-1 / 2$ then $\left|\frac{\lambda}{\lambda+1}\right|<1$ and thus $\left|\frac{\lambda p}{\lambda+1}\right|<|a|,\left|\frac{\lambda p}{\lambda+1}\right|<|a+p|$. The hypothesis of (1.8) are satisfied and it can be found that both sides of (6.2) are equal to $\zeta\left(s, a-\frac{p \lambda}{\lambda+1}\right)$.

For $p=1 / 2$, we have used (6.2) and the relation $\zeta(s+j, a+1 / 2)=\zeta(s+j, a)-2^{s+j} \sum_{k=0}^{\infty} \frac{(-1)^{k}}{(2 a+k)^{s+j}}$. If $p$ is an integer, formula (6.4) follows from the following identities

$$
\begin{gathered}
\zeta(s, a+p)=\zeta(s, a)-\sum_{k=0}^{p-1} \frac{1}{(k+a)^{s}} \\
\sum_{j=0}^{\infty}\left(\frac{p \lambda}{(\lambda+1)(k+a)}\right)^{j} \frac{(s)_{j}}{j !}=\left(1-\frac{p \lambda}{(\lambda+1)(k+a)}\right)^{-s}, 0 \leq k \leq p-1 .
\end{gathered}
$$

Remark 5. In (5.1), since the support of the weight function $\phi(u)$ is $\left[0, M_{0}\right]$, the theory of the moments implies that the $n^{\text {th }}$ root of $\int_{0}^{M_{0}} u^{n} \phi(u) d u$ converges to $M_{0}$ when $n$ tends to infinity. Thus, the sequence $\left(A_{(n, \lambda n)}^{(p)}(s, a)\right)_{n \in \mathbb{N}}$ :

$$
A_{(n, \lambda n)}^{(p)}(s, a)=(-1)^{n+1} p^{(1+\lambda) n+1} \frac{(s)_{(1+\lambda) n+1}}{(n+\lambda n) !} \int_{0}^{M_{0}} u^{n} \phi(u) d u
$$

satisfies

$$
\begin{aligned}
& \lambda>0 \Rightarrow \lim _{n}\left|A_{(n, \lambda n)}^{(p)}(s, a)\right|^{1 / n}=\left|\frac{\lambda^{\lambda}}{(\lambda+1)^{\lambda+1}} \frac{p^{1+\lambda}}{a(a+p)^{\lambda}}\right|<1, \\
& \lambda=0 \Rightarrow \lim _{n}\left|A_{(n, 0)}^{(p)}(s, a)\right|^{1 / n}=\left|\frac{p}{a}\right|<1 .
\end{aligned}
$$


Corollary 1. For all $s \in \mathbb{C} \backslash \mathbb{Z}^{-} \backslash\{1\}$, then ([14],chap. 3):

$$
\begin{aligned}
\sum_{j=1}^{\infty} p^{j} \frac{(s)_{j}}{j !} \zeta(s+j, a) & =\sum_{k=0}^{p-1}(k+a-p)^{-s},(p \in \mathbb{N}, \Re(a)>0,|a| \geq p), \\
\left(1-2^{1-s}\right) \zeta(s) & =\Sigma_{k \geq 1} \frac{(s)_{k}}{k !} \frac{\zeta(s+k)}{2^{s+k}}, \\
\Sigma_{k \geq 2} \zeta(k, 2) & =1, \\
\Sigma_{k \geq 2} \frac{k-1}{2^{k}} \zeta(k, 2) & =\frac{\pi^{2}}{8}-1, \\
\zeta(s) & =1+\frac{1}{s-1}-\Sigma_{k \geq 1} \frac{(s)_{k}}{(k+1) !} \zeta(s+k, 2) .
\end{aligned}
$$

Proof. The first formula follows from $\lambda \rightarrow \infty$ in (6.4). Then, in (6.3) $a=1$ and $\lambda \rightarrow \infty$ gives (6.6) and $s=1, p=1, a=2$ in (6.5) gives formula (6.7). (6.8) follows from the values $\lambda \rightarrow \infty, s=2, a=2$ in (6.3).

To obtain formula (6.9), we have to divide formula (6.4) by $s$, replace $s$ by $s-1$ and set $\lambda \rightarrow$ $\infty, a=2$ and $p=1$.

For alternated sums, we have ([14], chap.3):

Corollary 2. For all $s \in \mathbb{C} \backslash \mathbb{Z}^{-} \backslash\{1\}$, then:

$$
\begin{aligned}
& \sum_{j=1}^{\infty}(-1)^{j+1} p^{j} \frac{(s)_{j}}{j !} \zeta(s+j, a)=\sum_{k=0}^{p-1}(k+a)^{-s},(p \in \mathbb{N}, \Re(a)>0,|a| \geq p), \\
& \sum_{j=1}^{\infty}(-1)^{j+1} \frac{(s)_{j}}{j !} \zeta(s+j, a)=a^{-s},(\Re(a)>0,|a| \geq 1), \\
& \zeta(s, a)=a^{-s}\left(\frac{1}{2}+\frac{a}{s-1}\right)+\frac{1}{2} \Sigma_{k \geq 1} \frac{(-1)^{k-1} k(s)_{k+1}}{(k+2) !} \zeta(s+k+1, a),(\Re(a)>0,|a| \geq 1) .
\end{aligned}
$$

Proof. If we set $\lambda=0$ in (6.4) then $\sum_{j=1}^{\infty}(-1)^{j+1} p^{j} \frac{(s)_{j}}{j !} \zeta(s+j, a)=\sum_{k=0}^{p-1}(k+a)^{-s}$.

The value $p=1$ in the previous formula leads to (6.11).

If we substitute $s$ by $s-1$, in (6.11), and after dividing by $(s-1)$ we obtain

$$
\sum_{j=1}^{\infty}(-1)^{j+1} \frac{(s)_{j-1}}{j !} \zeta(s-1+j, a)=\frac{a^{-s+1}}{s-1} .
$$

Formula (6.11) follows from the addition of the previous formula with the following identity $\sum_{j=1}^{\infty}(-1)^{j+1} \frac{(s)_{j}}{j !} \zeta(s+j, a) \frac{1}{2}=\frac{a^{-s}}{2}$.

\section{Lacunary recurrence relations}

If in (6.3), (6.4), we let $\lambda=1$, then only $\zeta$ - values with odd index are involved and we get: 
Corollary 3. For all complex number s different from $0,-2,-4, \ldots$, then ([14], chap. 4):

$$
\begin{aligned}
\sum_{\substack{j \text { odd } \\
j \geq 1}}^{\infty} 2^{1-j} p^{j} \frac{(s)_{j}}{j !} \zeta(s+j, a) & =\sum_{k=0}^{p-1}\left(k+a-\frac{p}{2}\right)^{-s},(p \in \mathbb{N}, \Re(a)>0,|a| \geq p), \\
\sum_{\substack{j \text { odd } \\
j \geq 1}}^{\infty} 2^{1-j} \frac{(s)_{j}}{j !} \zeta(s+j, a) & =\left(a-\frac{1}{2}\right)^{-s},(\Re(a)>0,|a| \geq 1), \\
\sum_{\substack{j \text { odd } \\
j \geq 1}}^{\infty} \frac{(s)_{j}}{j !} \frac{\zeta(s+j)}{2^{j}} & =2^{s-1} .
\end{aligned}
$$

Proof. The first formula is obtained by setting $\lambda=1$ in (6.4). Then, the next formulas are only particular cases of the first one with $p=1$ for $(7.2)$, and $p=1, a=1$ for (7.3).

The following lacunary formulas seem to be new:

\section{Corollary 4.}

$$
\begin{aligned}
\sum_{k=0}^{\infty}\left(-\frac{1}{4}\right)^{k} \zeta(4 k+3) & =1, \\
\sum_{k=0}^{\infty}\left(-\frac{1}{4}\right)^{k}(4 k+3) \zeta(4 k+4) & =2 .
\end{aligned}
$$

Proof. If we take $\lambda=i\left(i^{2}=-1\right)$ in formula (6.4), with $p=1$, we obtain

$$
\sum_{j=1}^{\infty} \frac{i^{j}-(-1)^{j}}{(i+1)^{j}} \frac{(s)_{j}}{j !} \zeta(s+j, a)=\left(a-\frac{i}{i+1}\right)^{-s} .
$$

Equating imaginary part for the two handsides gives

$$
\sum_{j \equiv 2(4)}^{\infty}\left(-\frac{1}{4}\right)^{\frac{j-2}{4}} \frac{(s)_{j}}{j !} \zeta(s+j, a)=\Im\left(a-\frac{i}{i+1}\right)^{-s},
$$

and $a=1, s=1$ and $s=2$ in (7.6) implies Corollary 4

\section{An arithmetical property}

In this section we restrict ourselves to the case $m=\lambda n \in \mathbb{N}, p=1, a=1, s \in \mathbb{N} \backslash\{0,1\}$.

Theorem 6. For each integers $\lambda \geq 1, s \geq 2$, there exist sequence of integers $\beta_{0}(n),\left(\alpha_{j}(n)\right)_{0 \leq j \leq \lambda n}$ such that

$$
\lim _{n \rightarrow \infty}\left(\beta_{0}(n)+\sum_{j=0}^{\lambda n} \alpha_{j}(n) \zeta(s+j)\right)=0 .
$$

More precisely,

$$
0 \leq(-1)^{n+1}\left(\beta_{0}(n)+\Sigma_{j=0}^{\lambda n} \alpha_{j}(n) \zeta(s+j)\right) \leq \frac{(s+1)_{n+\lambda n}}{n !(\lambda n) !} M_{0}^{n}=O\left(2^{-\lambda n}\right),
$$

where $M_{0}:=\frac{2^{-\lambda} \lambda^{\lambda}}{(\lambda+1)^{\lambda+1}}$ 
Proof. Using Theorem 1 with $a=1, p=1$ and $m=\lambda n$, we can write

$$
\begin{aligned}
\sum_{j=0}^{\lambda n}\left(\left(\begin{array}{c}
n+\lambda n-j \\
n
\end{array}\right)-(-1)^{j}\left(\begin{array}{c}
n+\lambda n-j \\
\lambda n
\end{array}\right)\right) \frac{(s)_{j}}{j !} \zeta(s+j)-\left(\begin{array}{c}
\lambda n+n+s \\
\lambda n
\end{array}\right)=\left(\begin{array}{c}
n+\lambda n \\
n
\end{array}\right) A_{(n, \lambda n)}^{(1)}(s, 1) \\
\text { with } A_{(n, \lambda n)}^{(1)}(s, 1)=(-1)^{n+1} \frac{(s)_{(1+\lambda) n+1}}{(n+\lambda n) !} \int_{0}^{1} x^{\lambda n}(1-x)^{n} \zeta(s+\lambda n+n+1,1+x) d x \\
\left.=(-1)^{n+1} \frac{(s)_{(1+\lambda) n+1}}{(n+\lambda n) !} \int_{0}^{M_{0}} u^{n} \phi(u) d u . \text { (See }(5.1)\right) .
\end{aligned}
$$

Since $\phi$ is positive on $\left[0, M_{0}\right]$,

$$
\begin{aligned}
0 & \leq(-1)^{n+1} A_{(n, \lambda n)}^{(1)}(s, 1) \leq \frac{(s)_{(1+\lambda) n+1}}{(n+\lambda n) !} M_{0}^{n} \int_{0}^{M_{0}} \phi(u) d u=-\frac{(s)_{(1+\lambda) n+1}}{(n+\lambda n) !} M_{0}^{n} \frac{1}{s} A_{(0,0)}^{(1)}(s, 1) \\
& =\frac{(s)_{(1+\lambda) n+1}}{(n+\lambda n) !} M_{0}^{n} \frac{1}{s}=\frac{(s+1)_{(1+\lambda) n}}{(n+\lambda n) !} M_{0}^{n} .
\end{aligned}
$$

Then, using Stirling formula,

$0 \leq(-1)^{n+1}\left(\begin{array}{c}n+\lambda n \\ n\end{array}\right) A_{(n, \lambda n)}^{(1)}(s, 1) \leq\left(\begin{array}{c}n+\lambda n \\ n\end{array}\right) \frac{(s+1)_{(1+\lambda) n}}{(n+\lambda n) !} M_{0}^{n}=\frac{(s+1)_{n+\lambda n}}{n !(\lambda n) !} M_{0}^{n} \sim \frac{n^{s}(1+\lambda)^{s+1 / 2}}{s ! \sqrt{2 \pi \lambda n}} 2^{-\lambda n}$.

The proof is achieved by setting

$$
\beta_{0}(n):=-\left(\begin{array}{c}
\lambda n+n+s \\
\lambda n
\end{array}\right) \in \mathbb{N}, \alpha_{j}(n):=\left(\left(\begin{array}{c}
n+\lambda n-j \\
n
\end{array}\right)-(-1)^{j}\left(\begin{array}{c}
n+\lambda n-j \\
\lambda n
\end{array}\right)\right) \frac{(s)_{j}}{j !} \in \mathbb{N} .
$$

Remark 6. If $\lambda=1$, then $\alpha_{2 k}(n)=0,(k \in \mathbb{N}), M_{0}=1 / 8$, and thus formula 8.1 is valid for $s \in \mathbb{N} \backslash\{0\}$. Then

$$
\begin{aligned}
0 & \leq(-1)^{n+1}\left(2 \sum_{k=1}^{(n+1) / 2}\left(\begin{array}{c}
2 n-2 k+1 \\
n
\end{array}\right) \frac{(s)_{2 k-1}}{(2 k-1) !} \zeta(s+2 k-1)-\left(\begin{array}{c}
2 n+s \\
n
\end{array}\right)\right)=(-1)^{n+1}\left(\begin{array}{c}
2 n \\
n
\end{array}\right) A_{(n, n)}^{(1)}(s, 1) \\
& \leq \frac{(s+1)_{2 n}}{8^{n} n !^{2}} \sim \frac{2^{s} n^{s-1 / 2}}{s ! \sqrt{\pi}} 2^{-n} .
\end{aligned}
$$

If $s=1$ (resp. $s=2$ ), we get a relation involving zeta values at even (resp. odd) integers:

$$
\begin{gathered}
0 \leq(-1)^{n+1}\left(2 \sum_{k=1}^{(n+1) / 2}\left(\begin{array}{c}
2 n-2 k+1 \\
n
\end{array}\right) \zeta(2 k)-\left(\begin{array}{c}
2 n+1 \\
n
\end{array}\right)\right) \leq \frac{(2)_{2 n}}{8^{n} n !^{2}} \sim \frac{n^{1 / 2}}{\sqrt{\pi}} 2^{-n+1} . \\
\Rightarrow \lim _{n \rightarrow \infty}\left(2 \sum_{k=1}^{(n+1) / 2}\left(\begin{array}{c}
2 n-2 k+1 \\
n
\end{array}\right) \zeta(2 k)-\left(\begin{array}{c}
2 n+1 \\
n
\end{array}\right)\right)=0,
\end{gathered}
$$

and

$$
\begin{gathered}
0 \leq(-1)^{n+1}\left(4 \sum_{k=1}^{(n+1) / 2}\left(\begin{array}{c}
2 n-2 k+1 \\
n
\end{array}\right) k \zeta(2 k+1)-\left(\begin{array}{c}
2 n+2 \\
n
\end{array}\right)\right) \leq \frac{(3)_{2 n}}{8^{n} n !^{2}} \sim \frac{n^{3 / 2}}{\sqrt{\pi}} 2^{-n+1} \\
\Rightarrow \lim _{n \rightarrow \infty}\left(4 \sum_{k=1}^{(n+1) / 2}\left(\begin{array}{c}
2 n-2 k+1 \\
n
\end{array}\right) k \zeta(2 k+1)-\left(\begin{array}{c}
2 n+2 \\
n
\end{array}\right)\right)=0 .
\end{gathered}
$$




\section{References}

[1] T. Agoh, Recurrences for Bernoulli and Euler polynomials and numbers, Expo. Math. 18 (3) (2000) 197-214.

[2] G. A. Baker, Jr., P. Graves-Morris, Padé approximants, vol. 59 of Encyclopedia of Mathematics and its Applications, 2nd ed., Cambridge University Press, Cambridge, 1996.

[3] C. Brezinski, Computation of Padé approximants and continued fractions, J. Comput. Appl. Math. 2 (2) (1976) 113-123.

[4] C. Brezinski, Accélération de la convergence en analyse numérique, Lecture Notes in Mathematics, Vol. 584, Springer-Verlag, Berlin, 1977.

[5] C. Brezinski, Padé-type approximation and general orthogonal polynomials, vol. 50 of International Series of Numerical Mathematics, Birkhäuser Verlag, Basel, 1980.

[6] C. Brezinski, A direct proof of the Christoffel-Darboux identity and its equivalence to the recurrence relationship, J. Comput. Appl. Math. 32 (1-2) (1990) 17-25, extrapolation and rational approximation (Luminy, 1989).

[7] C. Brezinski, Convergence acceleration during the 20th century, J. Comput. Appl. Math. 122 (1-2) (2000) 1-21, numerical analysis 2000, Vol. II: Interpolation and extrapolation.

[8] W. Y. C. Chen, L. H. Sun, Extended Zeilberger's algorithm for identities on Bernoulli and Euler polynomials, J. Number Theory 129 (9) (2009) 2111-2132.

[9] W. Gautschi, Orthogonal polynomials - constructive theory and applications, in: Proceedings of the international conference on computational and applied mathematics (Leuven, 1984), vol. $12 / 13,1985$.

[10] W. B. Jones, W. J. Thron, Continued fractions, vol. 11 of Encyclopedia of Mathematics and its Applications, Addison-Wesley Publishing Co., Reading, Mass., 1980.

[11] M. Prévost, Padé approximation and Apostol-Bernoulli and Apostol-Euler polynomials, J. Comput. Appl. Math.(2009), doi:10.1016/j.cam.11.050.

[12] M. Prévost, Acceleration of some logarithmic sequences, J. Comput. Appl. Math. 55 (3) (1994) 357-367.

[13] M. Prévost, A new proof of the irrationality of $\zeta(2)$ and $\zeta(3)$ using Padé approximants, J. Comput. Appl. Math. 67 (2) (1996) 219-235.

[14] H. M. Srivastava, J. Choi, Series associated with the zeta and related functions, Kluwer Academic Publishers, Dordrecht, 2001.

[15] D. V. Widder, The Laplace Transform, Princeton Mathematical Series, v. 6, Princeton University Press, Princeton, N. J., 1941.

[16] J. R. Wilton, A proof of Burnside's formula for $\log \Gamma(x+1)$ and certain allied properties of Riemannn's $\zeta$-function, Messenger Math. 52 (2) (1922) 90-93. 